\title{
Comments On "Electromagnetic Radiation And The Afterlife"
}

\author{
Steven M. Rosen, Ph.D \\ Department of Psychology, The College of Staten Island, \\ City University of New York
}

I found the article "Electromagnetic Radiation and the Afterlife" interesting and ambitious. Janusz Slawinski delves into an exceedingly difficult area of investigation. However, his paper contains three related problems. The first is empirical, the second philosophical, and the third a matter of mathematical principle.

Slawinski wishes to put consciousness, and the question of its survival following biophysical death, on a scientific footing. He attempts to do so by postulating that the conscious ego and its biological substrate derive from electromagnetic force-fields that "mold and control" all life. In reading the evidence presented, I find the claim of such a strong shaping influence not sufficiently supported, since to show, for instance, that "changes of the conformational state of deoxyribonucleic acid (DNA) macromolecules in the cell nucleus are associated Imy emphasis] with photon emission" is not to show direct causal determination. The author himself confirms the tentative status of his hypothesis by admitting that "Assumptions about the electromagnetic nature of consciousness and conservation of its information content during the dying process are conjectures based on preliminary experiments and theoretical considerations."

Now, in view of the limited character of the empirical evidence, if Slawinski were to soften his occasionally extravagant claims so that the speculativeness of his approach were frankly conceded, the claims might be acceptable as speculation. Another example of such a claim is

Dr. Rosen is Associate Professor of Psychology at the College of Staten Island. Requests for reprints should be sent to Dr. Rosen at the College of Staten Island, City University of New York, 715 Ocean Terrace, Staten Island, NY 10301. 
Slawinski's assertion that "It becomes evident from the brief considerations above that research on low-level luminescence and other radiations of living systems provide [sic] both ample information on fundamental life processes and a realistic basis for dealing with the problem of an afterlife." But this brings me to my other serious reservations. The philosophical problem is that, as physicist/philosopher David Bohm might say, the author commits the error of reducing meaning to information; Bohm has emphasized the vast distinction between these. Therefore, Slawinski views the conscious ego, with its deeply subtle shadings of motives, nuances of intentions, feelings. thoughts, and intuitions, as a simple array of electromagnetically coded information stored in the body. Accordingly, he asserts that the contents of information (that is, meanings) may be "attained" by modulation of an underlying "carrying electromagnetic field." I believe philosophers like Stephen Braude, in the context of critiquing the identity theory of brain/mind interaction, have effectively exposed the fallacy of contending that one can read the contents of consciousness from biophysical brain activity. It appears that such naive reductionism, confusing two fundamentally distinct and incommensurable categories of being as it does, may be taken as a species of what Alfred North Whitehead called the fallacy of the misplaced concrete: the abstractive externalizations of the intellect are mistaken for the lived concreteness of experiential immediacy.

Finally, I feel there is significant confusion at the level of mathematical/physical principle. The author associates timelessness with the time dilation effect of Einstein's special theory of relativity. Only in passing does he acknowledge that this timelessness is merely metrical, not topological. But as I understand it, the retention of topological time would preclude attainment of the profound order of timelessness inherent to philosophies like Taoism, which Slawinski erroneously views as compatible with his own approach; nor could we square the maintenance of topologically continuous time with the notion that life does not last at all; it just is. A radical timelessness of this sort, which might be associated with accounts of the afterlife, near-death experiences, mystical states of awareness, and so forth, is characterized by a sense of thoroughgoing, unmitigated unity. However, the collapse of the metrical scale notwithstanding, it is the preservation of topological continuity that imposes spatiotemporal disjunctions (that is, disunity) quite out of keeping with the possible afterlife experience that life "just is." The confusion becomes evident when Slawinski apparently correlates the experience of timelessness with topological connectedness. What seemingly is not appreciated is that topological connectedness is 
merely a global feature that conserves local separation. It can convincingly be demonstrated that for the "instant information" transfer of which Slawinski speaks, a nontopological, or metatopological, connectedness would be required that would take us to the frontiers of Einstein's general theory of relativity, and to the foundations of quantum physics. In my opinion, this is where the action is, as far as the nexus of consciousness with the physical world is concerned. And it is precisely here that reductive notions of consciousness as an array of information/energy occupying physical space are called deeply into question. Indeed, the attempt to represent consciousness as an entity extended in the physical continuum can be seen as none other than an effort to express it in terms of topological continuity. So in the final analysis, the philosophical and mathematical objections are intimately related. 